\title{
eXSRUP: Hybrid Software Development Model Integrating Extreme Programing, Scrum \& Rational Unified Process
}

\author{
Nidhi Sharma, Manoj Wadhwa \\ Department of Computer \& Science, Echelon Institute of Technology, Faridabad, India \\ Corresponding author, e-mail: manojkw@gmail.com,nim.nidhi23@gmail.com
}

\begin{abstract}
Software industries are progressively adopting the agile development practices of customized models such as Extreme Programming (XP) or Scrum or Rational Unified Process (RUP). Scrum and Extreme Programming $(X P)$ are frequently used agile models, whereas Rational Unified Process (RUP) is one popular classic plan driven software development methodology. Both agile and plan driven models have their own merits \& demerits such as XP has good engineering practices, team collaboration and on the other hand weak documentation, poor performance in medium \& large scale projects. Scrum is based on project management practices. RUP model has some limitations such as impractical for small and fast paced projects, tendency to be over budgeted, condemn rapid changes in requirements. This research paper based on proposes hybrid framework eXSRUP by combining strengths of Scrum, XP and RUP by suppressing their limitations to produce high quality software.
\end{abstract}

Keywords: extreme programming (XP), scrum, rational unified process (RUP), XP scrum RUP (eXSRUP)

Copyright $\odot 2015$ Institute of Advanced Engineering and Science. All rights reserved.

\section{Introduction}

Extreme Programming $(\mathrm{XP})$ is the most highly adopted agile practice and widely used in various organizations and software industry throughout the world. XP is simple \& lightweight agile methodology for small scale \& simple projects. XP believe on basic five working codes/values are communication, simplicity, feedback, courage and respect. XP is designed for small teams who need to work in a fast \& quick software development environment, where requirements are changing frequently \& exceptionally. XP works by bringing the whole team together in the presence of simple practices, with continuous feedback to enable the project team to see their position. XP is resource oriented rather than process centric. Its follows an iterative and incremental approach; highly focusing on regular customer collaboration, embracing changes anytime, anywhere. Releases are delivered via small iterations, minimum error level. It's also prioritizing the project artifacts and work on the task with high level priority. Handling the rapidly changing business requirements is main capability of XP. Due to direct customer involvement by giving constant feedback, XP has a positive impact on the business requirements, which producing high quality software.

According to customer desires. XP main strengths are include rapid development, low cost, high quality, result oriented development, small bug rates and embracing of rapid changes at any stage with minimum possible expenses. Common XP practices are include Planning Game, Small Releases, Metaphor, Simple Design, Tests, Refactoring, Pair Programming, Collective Ownership, Continuous Integration, 40-hour Week On-site customer and Coding Standards [1-6].

Scrum is a popular and widely adopted agile software development technique/model. Scrum is focus on project leadership and some aspects of requirements management, which is derived from best business practices in terms of productivity and quality. Scrum is a lightweight framework and is suitable to integrate with other iterative incremental models work on complicated projects. Scrum also has the ability to promote the existing business practices, which increase the quality as well as productivity of the projects.

The iteration in Scrum is called sprint, which is more suitable for distributed teams of project initialization. Sprint is (2-6 week) time boxed or iterations. In some projects requirements 
are unclear \& ambiguous in this situation Scrum development methodology is the best practice. Scrum speeding up development, objectives alignment, creative business culture, promote share-holder value \& promoting individual improvement. Scrum helps to a software providers \& vendors to compete with others to achieve the market value. The main objective of Scrum development to manage the development processes of system with such practice to deliver high quality software. Scrum promoting self-organizing teams and helps to provide productive flexible working environment. It is an incremental and iterative process technique that conducting continuous communication meetings which are highlighting the overlapping areas, module integration \& data validation. The sprint or time box length in scrum usually from two to four weeks which can helps to finish the project within few months [3], [7-10], [21].

The Rational Unified Process (RUP) is an incremental, iterative and plan oriented architectural framework, focusing on standard software engineering principles. It is a step by step process methodology to promote qualitative object oriented software projects. RUP is a conventional \& plan driven approach, which provides a very clear structured and formalized flow for software development. RUP based on planning centric process, extensive system analysis, proper design principles, standard coding process and extended level of documentation. RUP are suitable for large scale projects due to extensive documentation, case driven, predictability, best assurance, tailoring and tool support processing. RUP can also be customized and tailored eXSRUP: Hybrid Software Development Model Integrating XP, Scrum \& RUP.According to business requirements in mid-level projects [11-12], [20-21].

The core idea behind this study is to propose a hybrid model (eXSRUP) to combine the best practices \& depressed limitations of existing agile models (XP, Scrum \& RUP) to increase the capability of software industry to produce high quality in the software projects on time and within budget.

eXSRUP (XP, Scrum \& RUP) model is to develop integration among Extreme Programming (XP), Scrum \& Rational Unified Process (RUP), while the focus of XP to provide very effective engineering practices, Scrum main goal to provide effective framework for management of the project and Rational Unified Process (RUP) is a conventional model, which believe on documentation and plan oriented. This integrated hybrid model of XP, Scrum and RUP will combine their best practices to achieve the goal of satisfying business and customer needs. The resultant outcome of this integration will be a rich productive and an efficient model i.e., eXSRUP that having best engineering \& management practices of software engineering and more productive [13-15], [22, 25].

The proposed novice hybrid model i.e. eXSRUP will be the collaborative container for combining the best practices and strengths of XP, Scrum and RUP, such as XP providing the best software engineering practices, Scrum offering a best project management features and RUP directions are business objectives accomplishment and customer satisfactions. The proposed hybrid model is to combine the good features of XP, Scrum and RUP and to decrease their pitfalls to provide a qualitative software development model to ensure business needs and embrace changes smartly. eXSRUP (XP, Scrum and RUP) is intended to embed the management features from Scrum, coding and standard strengths from XP and business objective accomplishment \& customer satisfactions from RUP. The main logic behind creating eXSRUP model is to have a development methodology that has the capabilities to produce high quality products and low bug rate. This paper is organized as follows: section 2 will discuss, why do we need hybrid model eXSRUP? Section 3 will explore proposed hybrid model eXSRUP; and finally section 4 will conclude with discussion along with future direction.

\section{Proposed Hybrid Software Development Model eXSRUP}

As described above agile practices are not well tested for large scale projects, but analysts are claiming that complex \& large size project can take advantages of them. To overcome this issue it is to build a hybrid model by combining leading agile methodologies, such as XP, Scrum and RUP. The eXSRUP Hybrid framework support to and extended the disciplines and principles of the agile manifesto. Project teams that are using iterative or incremental or agile processes have to produce high quality software, with higher return on investment (ROI), stakeholder satisfaction, and rapid deliver as compared to a conventional process model or an ad-hoc approach. By using some techniques such as refactoring, continuous integration (Cl), test-first development (TFD), and developer regression testing

TELKOMNIKA Vol. 16, No. 2, November $2015: 377-388$ 
(DRT) high quality can achieve. Return on investment could increase by focusing primary value activities with prioritize order, self-management, automation of regular activities, close collaboration etc., $[14,18]$.

eXSRUP is the conceptualize model of many techniques and principles from the three most popular agile methodologies i.e. XP, Scrum \& RUP. Mostly eXSRUP practices are taken from the agile community, such as daily meetings, continuous integration $(\mathrm{Cl})$, and refactoring. The eXSRUP is hybrid process model/framework, which could adopt and tailors techniques \& practices from a different of sources. The eXSRUP model/framework is integrated form of the below methods [18].

\subsection{Extreme Programming (XP)}

eXSRUP inherited the strategies of XP, but not limited collective ownership code, refactoring, TDD (test driven development), $\mathrm{Cl}$ (continuous configuration), and others.

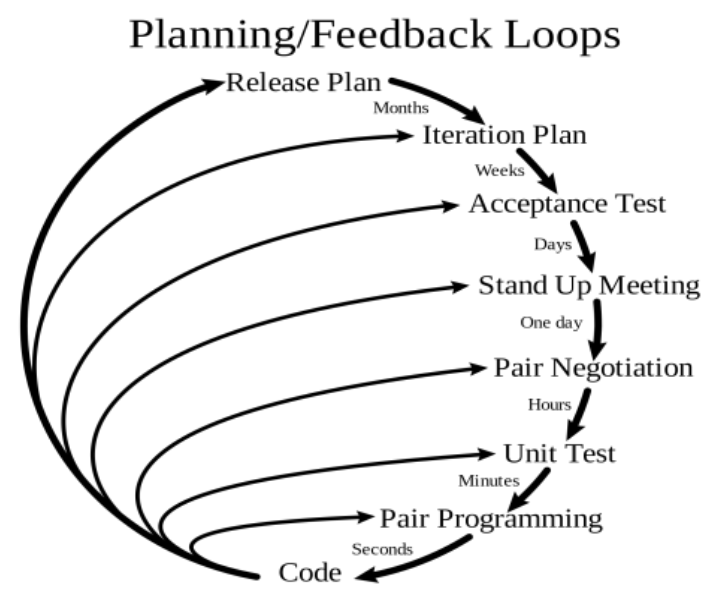

Figure 1. Extreme Programming process

\subsection{SCRUM}

Scrum primary focus is on management of requirements, guidelines and leadership. eXSRUP tailors many ideal things from Scrum and ignoring many of the scrum practices as well. eXSRUP adopting the idea of prioritize items, product ownership representative role, and a working potential deliverable is expected from each iteration; however, eXSRUP denounce some Scrum ideas and terminologies, such as scrum master no longer in use called as product owner, no speedy sprints etc.

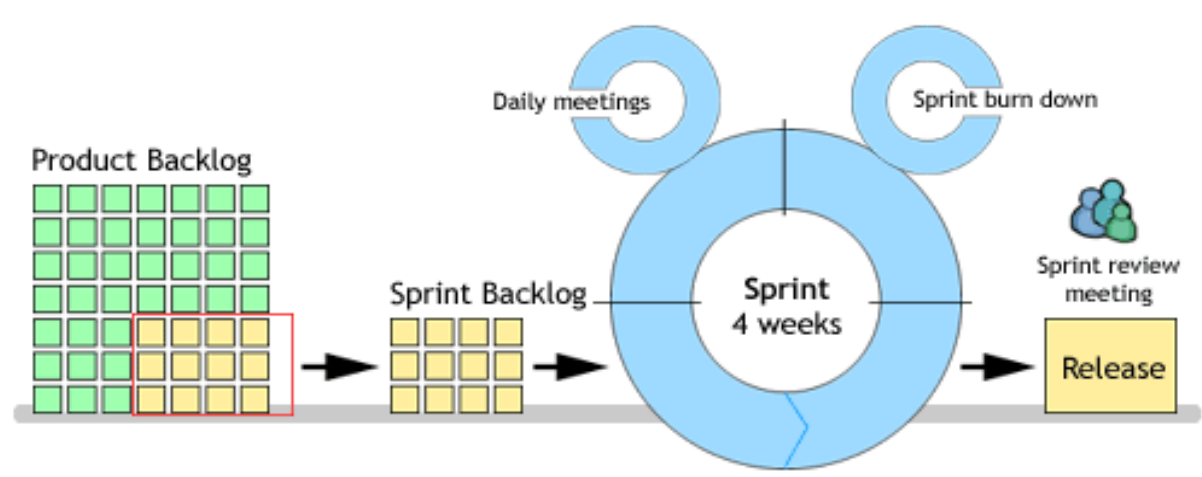

Figure 2. Scrum process 


\subsection{Unified Process (RUP)}

eXSRUP process framework is tailoring many ideas and strategies agile unified processes such as Open Unified Process (OUP) and Agile Unified Process (AUP). These strategies include explicit phase \& lightweight milestones and also inheriting the features of providing architecture and eliminating risks in initial iteration.

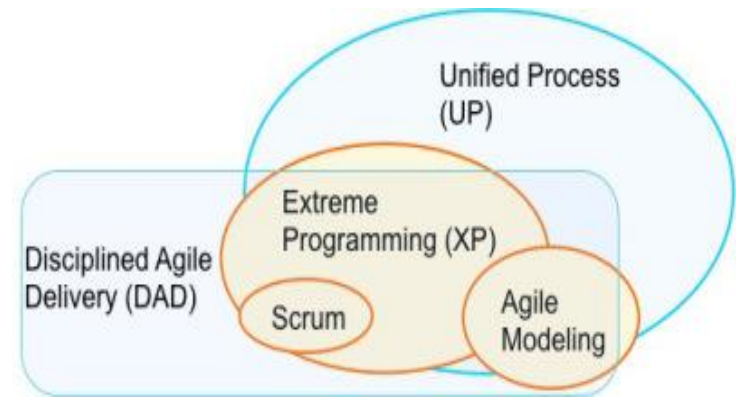

Figure 3. Comparison of XP, Scrum \& RUP in eXSRUP

eXSRUP provides a proper lifecycle of the project such as initialization, construction and releasing to end user. eXSRUP is recommending that each iteration is not same, but can evolve depends on change in project requirements along the lifecycle. eXSRUP believe in simplicity therefore distribute the project into phases, which each of them having lightweight milestones to focus on doing rights on right time with proper direction. These phases include initial visioning, architectural modeling, risk management, and deployment planning. This life cycle has several critical features:

\subsubsection{Delivery Lifecycle}

eXSRUP lifecycle extends of Scrum lifecycle, which are tends to show explicitly the complete delivery lifecycle from the initialization of a project to the release into production.

\subsubsection{Explicit Phases}

The eXSRUP lifecycle is consist of three phases such as inception phase, construction phase, and transition phase, reflecting the agile C3 (Coordinate Collaborate Conclude) cycle, as shown in Figure-2 below. [18]

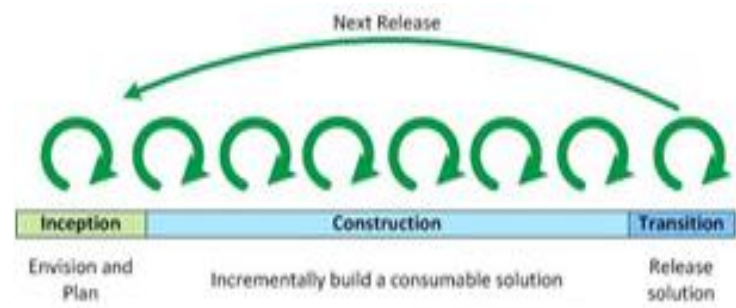

Figure 4. eXSRUP lifecycle

\subsubsection{Explicit Milestone}

eXSRUP framework contains a variety of milestones, which are playing an important role in governance and eliminating risks in projects. Let's overview the eXSRUP phases to better understand the contents of the eXSRUP process framework [18].

\section{a) The Inception Phase}

The primary objectives of the inception phase are to 1) identify the business scope of the new system and $A$ the project, 2) develop preliminary cost and schedule estimates based on the stakeholder concurrence, 3 ) identify the business need for the project, 4) understand the 
requirements according to the business case for the project, and 5) establish a vision for the solution. The business modeling discipline is highly utilized in the inception phase. The main activities of the business modeling discipline in this phase include: 1) create a list of business benefits, system objectives and system capabilities, 2) describe the problem or need, 3) consider business process, workflow, and interfaces to other systems, and 4) analyze the various system stakeholders, existing system architecture, and

\section{b) The Construction Phase}

The main focus in this phase goes to coding and testing the software. All the system components and features including user interfaces, business logics, data access functions, and help functions are implemented according to the specifications designed in the previous phase. This phase should produce a releasable working system so that the system can be deployed during the next phase. This phase can include several iterations that continue the design and implementation of the system. In particular, for large projects, several construction iterations can be involved in an effort to break the project into small and manageable tasks. [18]

\section{c) The Transition Phase}

During the transition phase, the system is delivered into production and becomes available to end users. One or more iterations in this phase can involve end user training with a user's manual, beta testing to validate the system functions against end users' expectation, and corresponding modification and fine tuning. If all the requirements are satisfied, the development cycle is closed. In this figure shows the four phases but these three methodologies are integrated to each other these four phases are reduce in three phases, those are mention in above discussion. Elaboration phase is not used in three methodologies [18].

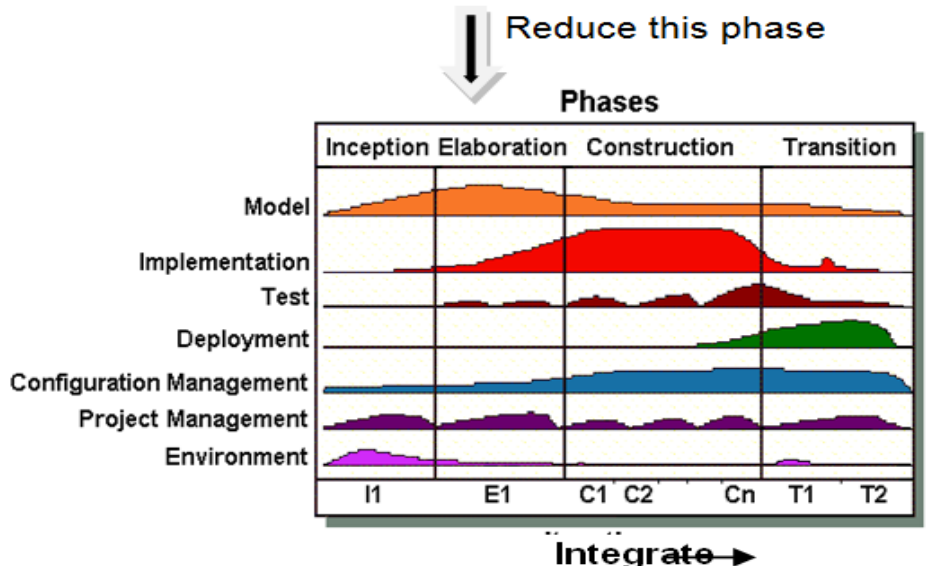

Figure 5. eXSRUP Process in different Phase

This is the first phase of our proposed model and it has 2 logical activities namely requirement gathering and pro- sect planning. We perform necessary tasks such as requirements gathering, project planning in this phase be- for starting the iterations because when the iteration is started then customer hardly has a chance to give feed- back in this phase (during the iteration) until he/she wants to change the overall project plan or project scope. Furthermore in this phase, it is finally decided how many iterations are needed in current project.

Second Evolution phase consist of Analysis, Designing module.

Third Production phases of the iteration and consumes more time as compared to other two phases of iteration (initialization and maintenance). This phase ensures the development of test cases, development (coding) of modules/sub-systems according to user stories/requirements and validation of modules/sub-systems using validation techniques to make sure that there is no bug and error in that module. 


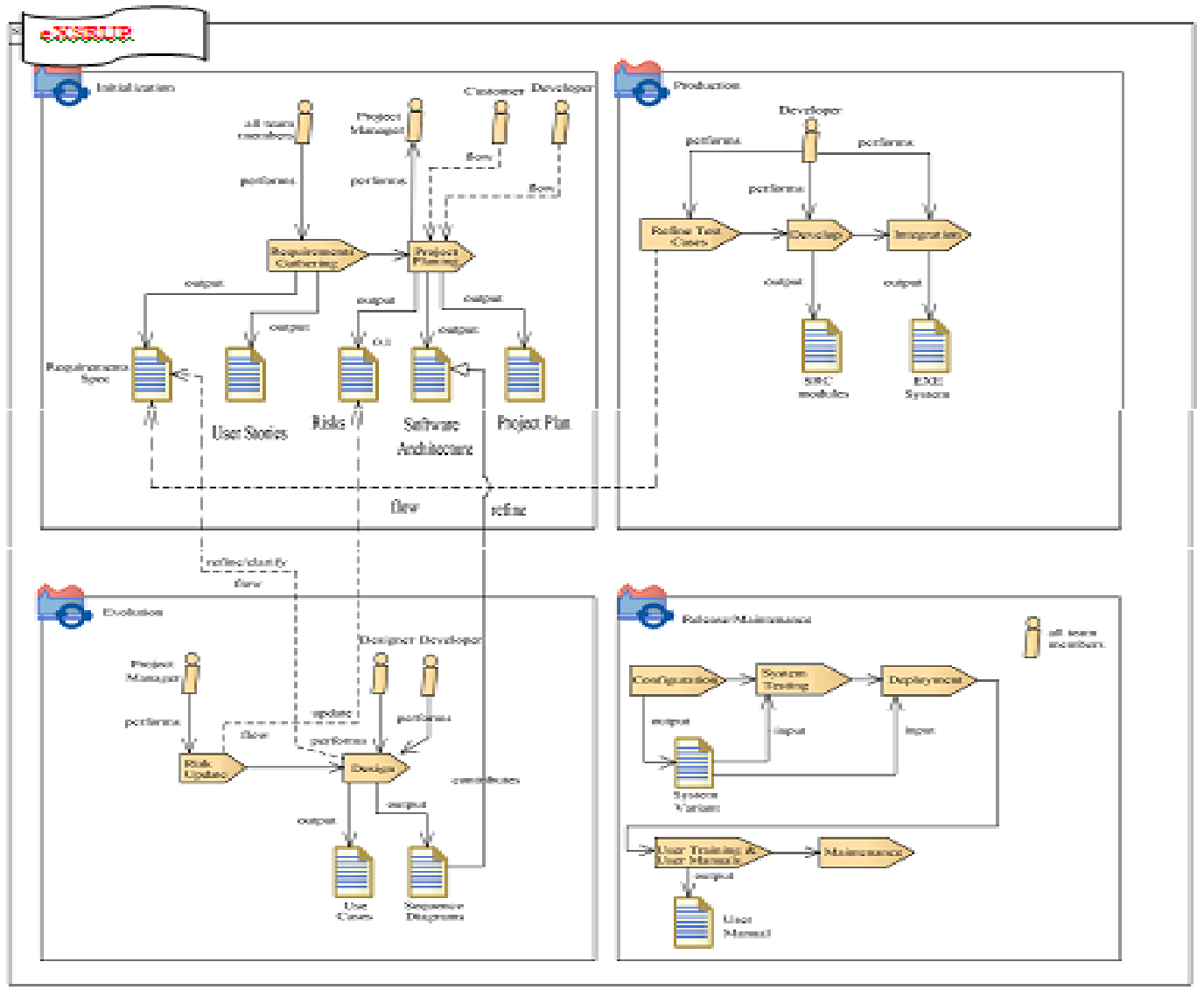

Figure 6. Architecture of proposed eXSRUP model

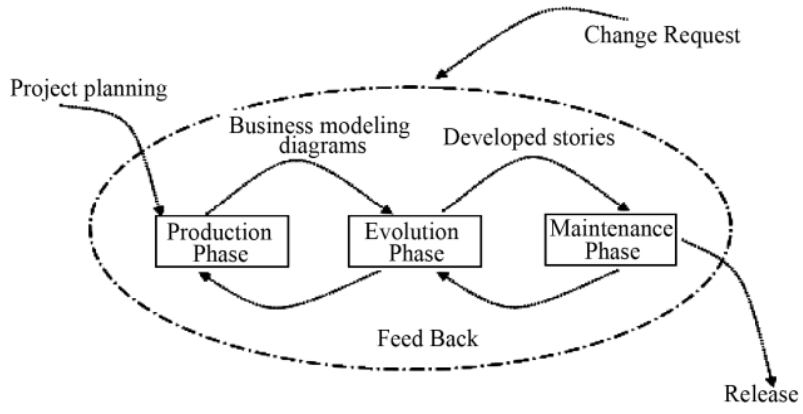

Figure 7. eXSRUP iteration cycle

Maintenance Phase this is last step of eXSRUP iteration cycle and consumes more resources than evolution phase and fewer resources than production phase. In this phase, team have to man- age the system which has been released to the customer and also take care of the integration with the previously developed/released module as well as integration testing of the product. Release phase consist of deployment, user training \& user manuals.

\section{Why do we need eXSRUP?}

Different agile models are experienced and practiced by integrating them with plan driven conventional software development models to increase the throughputs of both agile and classic models, while trying to suppress the pitfalls and limitations of each approach. Integration of Scrum, XP and RUP methodologies is a good combination to enrich the practices of both 
pompous and agile approaches. XP focus on engineering practices \& coding standards, but lacks in project management expertise; XP practices can apply in small projects due fully dependent on customer, which increase the project fail risks. On the other hand Scrum is focusing on project management practices and silent about the software engineering processes. Scrum required sound technical qualified resources to build the team. RUP model also ringing some risks \& limitations, such as over budgeting, rapid changes of requirements are getting slow response, suitable only for medium \& large scale projects rather than for fast paced and small scale projects. RUP model major pitfalls are that they are not providing proper guidelines for implementation of projects and leaving entirely on the user end. The best practices of Scrum and XP are to embrace rapid changes in requirements intended to add into eXSRUP model, while RUP getting fail in adaptation to frequent changes in requirements due to base on extensive system requirements documentation. The primary potency of RUP model is to meet business requirements \& customer satisfaction by delivering software with high quality and providing adequate planning of the system. Considering the context about XP, Scrum and RUP model, the research problem becomes "Need to propose a hybrid model by integrating the strengths of XP, Scrum \& RUP as well as narrower the flaws to build a quality software development model to adapt creeping requirements quickly with planning \& documentation" [3, 13], [15-16], [22].

eXSRUP is not a methodology, but it is generalized framework from which anyone can choose interested ideas, which might suitable for various organization or project. [14]. eXSRUP also has some extra structure that is not in some of agile models. Some interested points that trying to proof that framework is worthy [18]:

1) eXSRUP is an integrated framework of chairing agile models, combining together a set of gratuitous strengths such as Scrum, XP \& the Unified Process.

2) Existing agile models are mostly lacking of having practices about the full life cycle. For instance Scrum main focus on management oriented, rather than architecture centric. eXSRUP is a hybrid framework gleans heading strengths from full lifecycle.

3) eXSRUP a detonated recognition that mostly all enterprise level projects go through startup and deployment phases, which inception \& transition phases respectively.

4) eXSRUP believed on unbranded nomenclature such as "sprint" rather recommending common language words that is easy to understand instead of sticking with one's methodology preferences. eXSRUP is not means to replace any existing agile practice but to simplify \& promote them. Instead of saying that "I am working on project using Scrum, somehow XP, somewhere RUP practices etc.", simply can say that we are using the eXSRUP framework. For instance, if a team now doing Scrum, they could still say that they are following the eXSRUP framework. Using agile capabilities one can crazy about speedup or quality, or about additional scaling process; eXSRUP could help in these ideas, but only that it makes sense for project. In abstract, eXSRUP provides a comprehensive guidance with unbranded ideas that go beyond traditional agile models that help organization to deal enterprise in informal projects. [18]

\section{Case Study and Evolution}

The proposed eXSRUP model is validated through a con- trolled case study. The primary intention of conducting this case study was to develop same application by three different teams of students under the supervision of one project manager in the same environment. A Portal was developed using Mat lab. The source code of developed application cannot be publicized due to licensing issues. A training session was conducted before the start of each iteration. The total numbers of iterations is same in all the releases. The project team consists of four team members and total duration for each development was one month and one was the project manager in each team using four models and two programmers.

\subsection{Evaluation Parameters}

Software process models are evaluated for their usability and effectiveness on the basis of different parameters.

9 parameters which are important for evaluation of different process models. Table 1 depicts results of four releases of our case study developed using RUP, XP, Scrum and 
eXSRUP models. We filtered some parameters which were redundant such as lines of code and KLOC.

Results of Implementation:

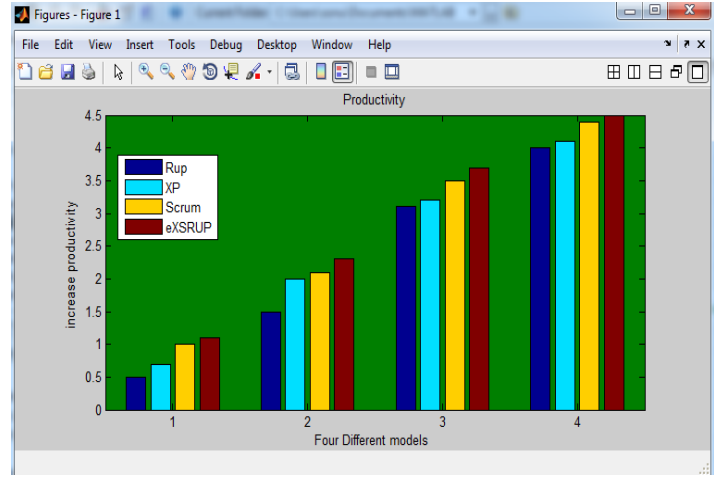

Figure 8. Productivity of each release in Rup, XP, Scrum, eXSRUP

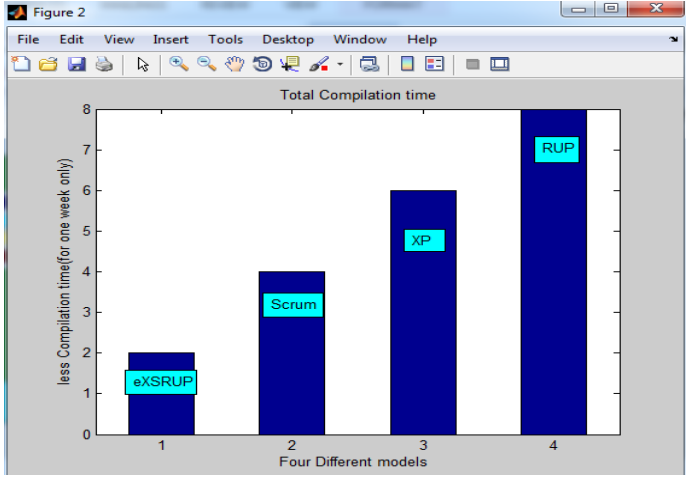

Figure 9. Total compilation time(week) of project in XP, RUP, Scrum and eXSRUP

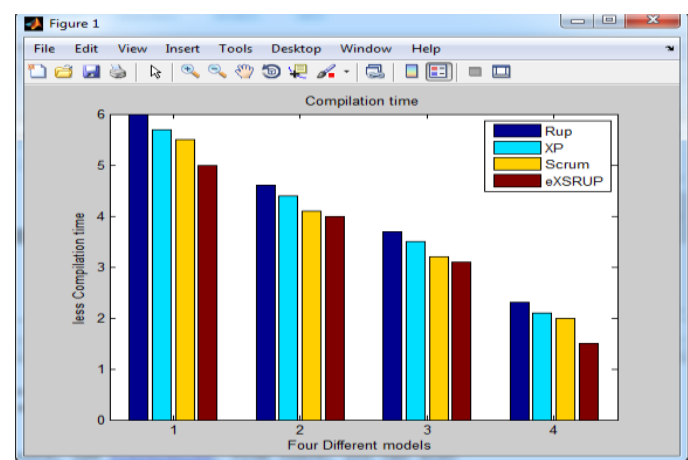

Figure 10. Compilation time (week) of each release in XP,RUP, Scrum and eXSRUP

Table 1. Evaluation results using four models

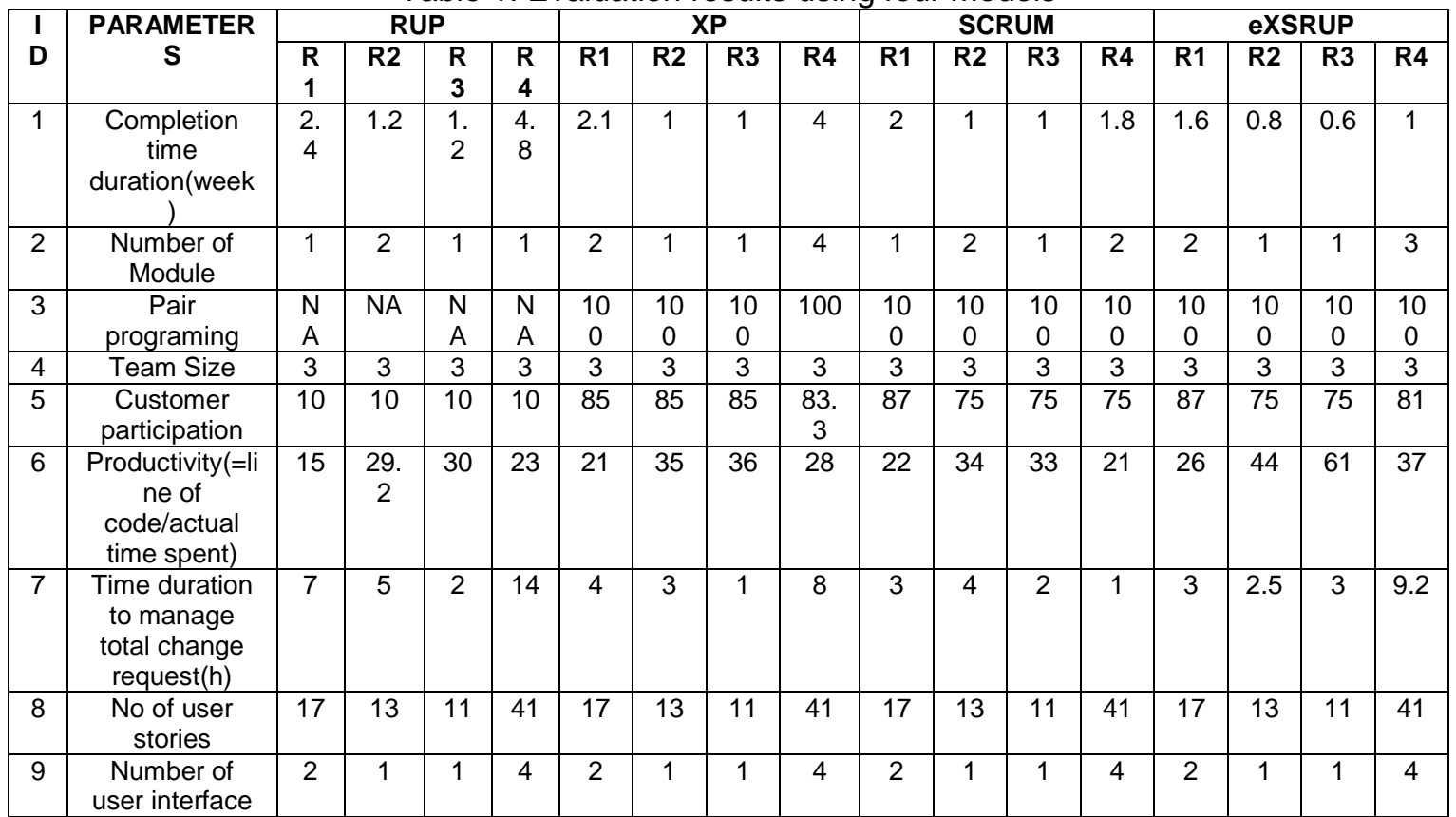

R1: Release; R2: Release; R3: Release; R4: Release

TELKOMNIKA Vol. 16, No. 2, November $2015: 377$ - 388 
Figure 8 graphically presents comparison of important parameters of model used for implementation of case study. The other parameters of eXSRUP can be seen in Table 1. It's very clear from Table 1 that our proposed model has significant improvement in all parameters which are used to evaluate the developed case study.

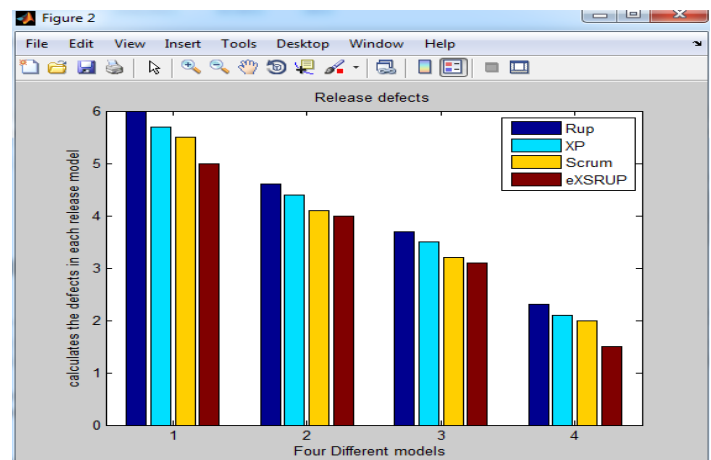

Figure 11. Post release defects of each release in RUP, XP, Scrum, eXSRup

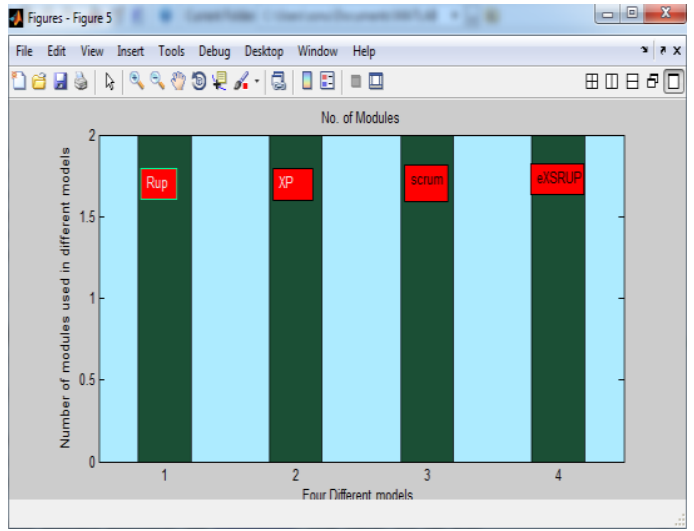

Figure 13. Used number of modules in each release in RUP, XP, Scrum, eXSRUP

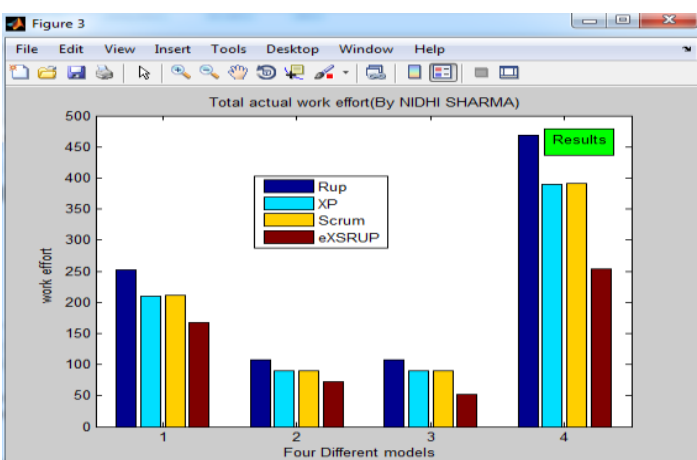

Figure 12. Total actual work release in each release in RUP, XP, Scrum, eXSRUP

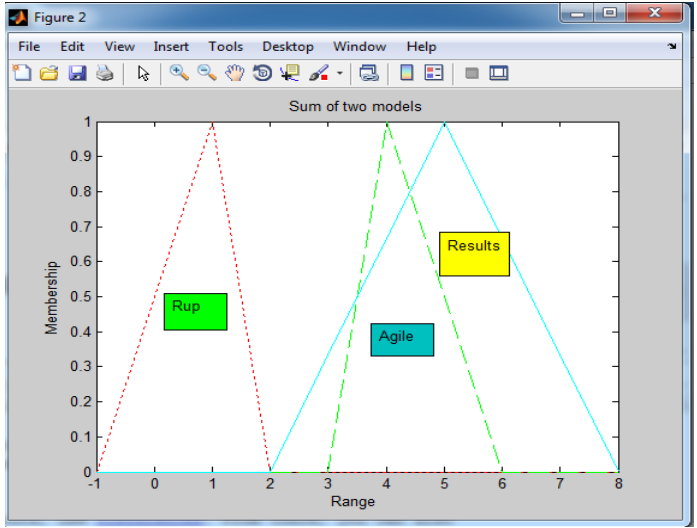

Figure 14. RUP \& Agile models sum produces high result

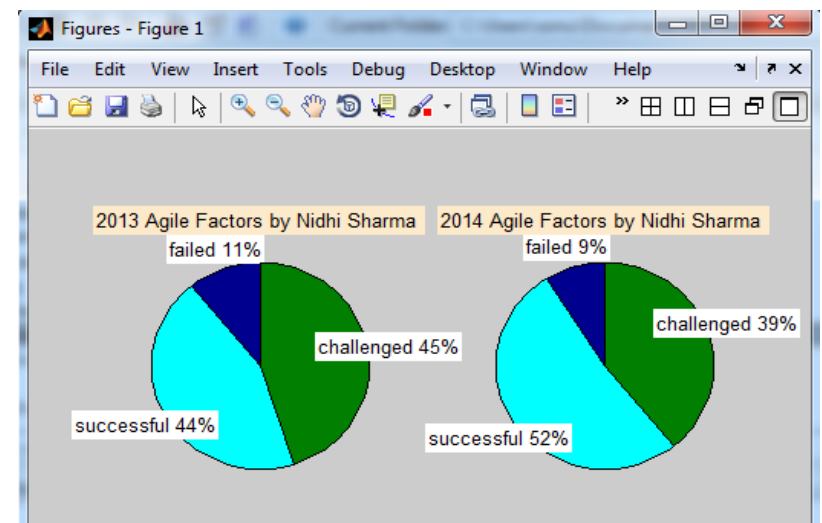

Figure 15. Results of Agile Factors

We do not claim same percentage of improvement for other type of systems until the proposed model is evaluated by the academia and industry. 
It is very difficult to compare results of presented model with other models because standard benchmark systems are not available for comparison. Different authors proposed integration of agile and conventional models and they evaluated performance of their models on different case studies. The nature of case studies vary in size, complexity, team structure, team size, team expertise, programming languages, tool support, customer requirements and various other factors. Due to these varying factors we developed same case study using four models one by one and results presented in Table 1 reflect that our proposed model has significant improvement in productivity, it take less time to manage change requests per hour and same system was developed with less time as compared with XP, Scrum and RUP.

\subsection{Validity}

Validity is the key challenge for researchers and pr-actioners in conducting empirical research work. Validity for empirical research to be acceptable as a contribution to scientific knowledge, the researcher needs to convince related academia and industry conclusions drawn from an empirical study are valid. Threats to validity of our results can be classified as below.

\subsubsection{Internal Validity}

Internal validity is addressed more frequently in experimental studies. It is concerned with the consistency of the measurements, appropriate use of tools, and methods. The implementation of real case study using RUP, XP, Scrum and eXSRUP validated proposed model. Internal validity is also affected by experimental bias. The same application was developed by four different teams in the same environment to reduce this threat as already published case studies may have been developed with different intentions and environment conditions.

\subsubsection{External Validity}

Key concern while conducting external validity is whether the findings of the study can be generalized be- yond the sample for which they were derived. To avoid this threat, the case study, and other tasks planned to conduct case study are designed keeping in view the schedule and knowledge level of students. The proposed model is an amalgamation of best features of RUP and XP, Scrum which is validated through case study, but we cannot generalize the improvement in productivity and other attributes until it should be evaluated on different types of systems by academia and industry. Currently, we are working on different other projects using our proposed model which will evaluate threats to external validity.

\subsubsection{Construct Validity}

Construct validity involve the relation between theory and observation. The proposed model can be customized according to nature of software and customers' requirements. The construct validity is established when proposed model is related to both conventional (RUP) and agile model (XP, Scrum).

\subsubsection{Reliability Validity}

This reliability affects the replicability of our results. The proposed model is evaluated using standards parameters as used by other case studies. However, we cannot publish the implemented systems due to licensing issues.

\section{Conclusion and Future work}

This research primary aim is to get such generic software development model to develop qualitative software that should be on time, meet customer needs and boost team performance. eXSRUP framework combining the strengths of the three popular agile models such as XP, RUP and Scrum while suppressing their pitfalls. XP will provide software engineering practices such as user stories, pair programming, and test driven activities. Scrum is popular for managerial techniques due to observations; roles based approach and artifacts throughout lifecycle of project development. RUP will help in providing structured, skeleton and formalized guidelines throughout lifecycle and also help in support of XP practices via its philosophy. 
The proposed eXSRUP framework also need proper testing \& review like other customized agile models XP, Scrum, Lean, Kanban, and Crystal clear etc. in real practical environment to promote it. Various industries as well individual professionals are working, on that way to improve the practices of XP, Scrum and RUP to manage software development and delivery of projects. My suggestions lets help us in promoting this new hybrid framework further by evaluating it in various sized and types of projects and organizations in different business environments. eXSRUP practical implementation can address its shortcomings \& flaws and can improve it by sharing ideas of practical experiences. Some recommendations for future work for extension of the eXSRUP framework are [19]:

1) eXSRUP framework can be further integrated with other models and standards for getting best solution.

2) More case studies \& survey with agile as well business professionals involved in variety of projects and business could improve eXSRUP framework.

3) Real implementation of eXSRUP framework could support the improvement in the hybrid model.

The real applications of eXSRUP hybrid model would take time, because every practitioner is cautious to implement a virgin model or practice that has not proved yet. Best approaches and practices are gradually, but continuously improved by testing and evidence. The breadcrumb of the eXSRUP framework will only be realized after more application in real environment [19].

The results of case study proved that presented model has improved the productivity, performance, completion time and various other attributes as shown in Table 1, only with the exception of time duration to manage change requests. The improvement in productivity is $15 \%$ and completion time is reduced to $20 \%$ which is significant improvement. The proposed model will be evaluated on other types of medium size projects using different programming languages.

\section{Acknowledgements}

We would like to convey my thanks message to Dr. (Professor) Manoj Wadhwa. Their advices and support make us able to complete our work and also thankful to Echelon Institute of Technology for providing us all the facilities and services during our studies.

\section{References}

[1] Abrahamson P, Salo O, Ron K. Agile Software Development Methods: Reviews and Analysis. VTT Electronics. 2002.

[2] A Sillitti, G Succi. The Role of Plan-Based Approaches in Organizing Agile Companies. Cutter IT Journal. 2006; 19(2): 14-19.

[3] Brent Barton, Evan Campbel, Ken. Implementing a Professional Services Organization Using Type C Scrum. In Proceedings of the 40th Annual Hawaii International Conference on System Sciences. Hawaii. 2007: 275.

[4] Extreme Programming Official Website. http://www.extremeprogramming.org/map/project.html.

[5] Ghulam Rasool, Shabib Aftab, Shafiq Hussain, Detlef Streitferdt. eXRUP: A Hybrid Software Development Model for Small to Medium Scale Projects. Journal of Software Engineering and Applications. 2013.

[6] Gul Ahmad, Tariq Rahim Soomro, Mohammad Nawaz Brohi. Agile Methodologies: Comparative Study and Future Direction. European Academic Research. 2014.

[7] Jiang, Armin Eberlein. Towards a framework for understanding the relationships between classical software engineering and agile methodologies. In Proceedings of the 2008 international workshop on Scrutinizing agile practices or shoot-out at the agile corral. Germany. 2008: 9-14.

[8] Juyun Cho. A Hybrid Software Development Method for Large-Scale Projects: Rational Unified Process With Scrum. Issues in Information Systems. 2009: 2.

[9] J Grey. The development of a hybrid agile project management methodology. Potchefstroom Campus of the North-West University. 2011.

[10] Kamlesh V, Ahmad S. Evaluating Evolutionary Prototyping for Customizable Generic Products in Industry. M.S. Thesis. Ronneby, Sweden: School of Engg. Blekinge Inst. Tech; 2008.

[11] Lamia Nassif, Jessy, Nadine Ghanem, Pedro Maroun Eid. Extreme Programming. Software Engineering CSC 423 B - MWF 11-12. 2002.

[12] M Grant. Introduction to Extreme Programming. 
[13] Marchesi M, Mannaro K, Uras S, Locci M. Distributed Scrum in Research Project Management. In Proceedings of the 8th International Conference on Agile processes in software engineering and extreme programming. Como, Italy. 2007: 240-244.

[14] Maria P, Sadra D, Casper L. Distributed Agile Development: Using Scrum in Large Projects. In Proceedings of IEEE International Conference on Global Software Engineering. Bangalore, India. 2008: 87-95.

[15] M Salman Bashir, M Rizwan Jameel Qureshi. Hybrid Software Development Approach For Small To Medium Scale Projects: Rup, Xp \& Scrum. Sci.Int. Lahore. 2012; 24(4): 381-384

[16] P Kroll, P Kruchten. Rational Unified Process Made Easy: A Practitioner's Guide to the RUP. Boston: Addison Wesley. 2003.

[17] P Kruchten. The Rational Unified Process-an Introduction. $2^{\text {nd }}$ Edition. Addison-Wesley. 2000.

[18] Schwaber K, Beedle M. Agile Software Development with Scrum. USA: Prentice Hall. 2001.

[19] Sadaf Un Nisa, M Rizwan Jameel Qureshi. Empirical Estimation of Hybrid Model: A Controlled Case Study. I.J. Information Technology and Computer Science. 2012; 8: 43-50.

[20] Szalvay V. An introduction to agile software development. 2012.

[21] Scott W Ambler. Disciplined Agile Delivery: An introduction. 2011.

[22] Zaigham Mushtaq, M Rizwan Jameel Qureshi. Novel Hybrid Model: Integrating Scrum and XP. I.J. Information Technology and Computer Science. 2012; 6: 39-40. 